glow is merely a rather feeble development of the well-known nitrogen afterglow.

Research Laboratories,

H. G. JENKINS

J. N. BOWTELI.

General Electric Co., Ltd., Wembley.

R. W. Strong

Osram-G.E.C. Lamp Works, Hammersmith.

${ }^{1}$ Vegard, L., Kon. Akad. Wet. Amsterdam, 27 (1924)

2 Vegard, I., and Kvifte, G., Nature, 162. 967 (1948).

\section{Cathodo-Luminescence of Rare Earths in Coal Ash}

bx cathodo-Iuminescence I have detected ${ }^{1}$ the presence of rare earths in certain minerals in quantities too small to be detected by arc spectrographic analysis.

Samples of a number of Indian coals (from Darjeeling, Assam, Raniganj, Jharia and Hyderabad) were incinerated in thin layers in porcelain basins in an electric furnace at $400^{\circ} \mathrm{C}$. The presence of rare earths in the ashes so obtained could not be detected by arc spectrographic analysis with a current of 10 amp. and 220 volts, with an $E_{1}$ quartz spectrograph using pure carbon rods. Cathodo-luminescence was then tried. The coal ashes as obtained did not show any luminescence when excited by the cathode rays. But after mixing the ash with a sufficient quantity of calcium oxide, the cathode rays induced bright luminescence. This was caused by the presence of manganese acting as an activator in the presence of calcium oxide. When rare earths were also present in the coal ash, the manganese band was suppressed by the activating action of the rare earths. It was found that the best condition for excitation of the specimen was at a potential of 4,000 volts, the current in the tube being kept at 4 milliamp. The luminescence was then so bright that the time of exposure required for taking a spectrogram was 3-7 min. with the direct-vision spectrograph and 5-12 min. with the Fuess quartz spectrograph. Urbain's data for the different systems for rare-earth oxide in calcium oxide were taken as reference in identifying the linelike bands of the luminescence spectra of the specimen.

The rare-earth activators for luminescence in coalash were samarium, dysprosium, europium, erbium and probably terbium and neodymium. It was found that the luminescence of the ash from only a few samples of coal from Darjeeling, Assam and Hyderabad was caused by the presence of rare earths. Luminescence of all the ashes from samples of coal from Raniganj and Jharia was caused only by manganese. Some of the samples of ash contained rare earths in sufficient quantity completely to suppress the luminescence spectrum of manganese in the visible region. With smaller quantities of rare earths, both the rare earths and the manganese behaved as activators for luminescence. Those rare earths with even atomic numbers (samarium 62, dysprosium 66) were found to be the chief activators for luminescence.

Thanks are due to Prof. P. B. Sarkar, for helpful discussions, to Prof. M. N. Saha, for allowing me to use the spectrograph, and to the Director of the Geological Survey of India, for supplying the samples of coals.

Inorganic Chemistry Laboratory,

University College of Science.and Technology, Calcutta. Sept. 27.

${ }^{1}$ Mukherjee, B. Ind. J. Phys., 22, 221 (1948); 22, 305 (1948).

\section{A Correction to the Diameter Measurement of Diffuse X-Ray Diffraction Rings}

LATTICE parameter measurements of cold-worked metals are subject to an appreciable error if no provision is made for the lack of coincidence between the $K \alpha_{1}$ peak and the apparent peak, as measured, of the broadened doublet. This is indicated in the synthesized curves of the accompanying figure.

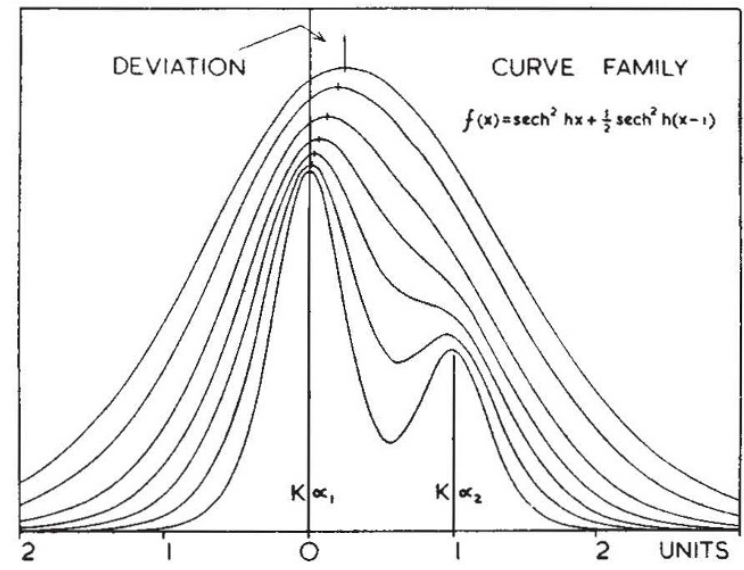

The geometrical subtraction method proving laborious, and in many cases uncertain, the following method of correction has been applied in recent work to obtain a better figure for lattice parameter. It depends upon a relationship determined between the width at half maximum intensity and the deviation between the $K \alpha_{1}$ and the apparent peak of the photometered doublet.

It was found that the practical doublets, when corrected for film response, approximated reasonably to the sum of two curves of the form

$$
y=\operatorname{sech}^{2} h x,
$$

the constant $h$ being typical of a particular degree of broadening.

The $K \alpha$ doublet was synthesized from two such curves, having an intensity ratio of $2: 1$ and a unit $K \alpha_{1}-K \alpha_{2}$ separation. This sum curve was defined by the following equation:

$$
f(x)=\operatorname{sech}^{2} h x+\frac{1}{2} \operatorname{sech}^{2} h(x-1) .
$$

Differentiation provided the condition for the maximum of this curve:

$\operatorname{sech}^{2} h x \cdot \tanh h x=-\frac{1}{2} \operatorname{sech}^{2} h(x-1) \cdot \tanh h(x-1)$.

The sum curve (1) was plotted for a particular value of $h$, and the approximate abscissa for the peak estimated.

Open-scale plots were then made, for a small range of abscissa values about the approximate value, of $f^{\prime}(x)=\operatorname{sech}^{2} h x \cdot \tanh h x$ and $f^{\prime \prime}(x)=\frac{1}{2} \operatorname{sech}^{2} h(x-1)$ tanh $h(x-1)$. The intersection of these two lines gave the correct peak abscissa, with reference to the foot of the $K \alpha_{1}$ peak as origin. Substitution ir equation (1) provided the corresponding ordinate.

To determine the half-width, an estimate of thi positive and negative abscissa values was obtainer from the plotted sum curve, and, using form (1) 